\title{
Isolated Nail Pigmentation Associated with Chikungunya: A Hitherto Unreported Manifestation
}

\section{Archana Singal Deepika Pandhi}

Department of Dermatology, University College of Medical Sciences and Guru Teg Bahadur Hospital, University of Delhi, Delhi, India

\section{Established Facts}

- A host of mucocutaneous manifestations are known to occur in approximately $30-50 \%$ of patients with chikungunya infection. Skin pigmentation is one of the most commonly encountered lesions.

- Coexisting nail involvement has been reported by few authors in the form of black lunula, melanonychia, onychomadesis, and subungual hemorrhage.

\section{Novel Insights}

- Isolated nail pigmentation/melanonychia without skin lesions has not been reported previously with chikungunya.

\section{Keywords}

Nail disorder · Pigmentation · Chikungunya · Melanonychia

\section{Abstract}

Chikungunya (CHK) fever is a re-emerging RNA viral infection caused by the bite of infected Aedes mosquitoes which presents with a multitude of mucocutaneous manifestations, including a characteristic morbilliform rash, centrofacial pigmentation, aphthae-like lesions, and vesiculobullous lesions sparing mucosae. The majority of these lesions resolve spontaneously and do not require specific therapy. With regular and cyclical outbreaks in many countries for many decades, it is obligatory for dermatologists to be aware of the different skin lesions in the wake of early diagnosis and the need for conservative management. We report 2 cases from India developing isolated melanonychia with $\mathrm{CHK}$ fever.
\end{abstract}

(c) 2017 S. Karger AG, Basel

\section{KARGER}

(c) 2017 S. Karger AG, Basel

E-Mail karger@karger.com

www.karger.com/sad

\section{Introduction}

Chikungunya virus (CHKV) is a re-emerging viral infection prevalent in Asia, Africa, and the Indian subcontinent. It is caused by a RNA virus that belongs to the alphavirus genus of the family Togaviridae. The disease is transmitted to humans by the bite of infected Aedes mosquitoes; 2 species, namely Aedes aegypti and Aedes albopictus, are the responsible vectors in Asia [1]. India has been witnessing periodic outbreaks of CHKV infection in different geographic regions since 2005 after a quiescent period of almost 3 decades. CHKV infection is characterized by sudden onset of high-grade fever, disabling muscle and joint pain, and skin rash. Diagnosis is often clinical and can be confirmed by serological tests, such as enzyme-linked immunosorbent assay that shows the presence of IgM and IgG anti-CHK antibodies. The diag- 
Fig. 1. Greyish brown pigmentation of the fingernails in patient 1 .

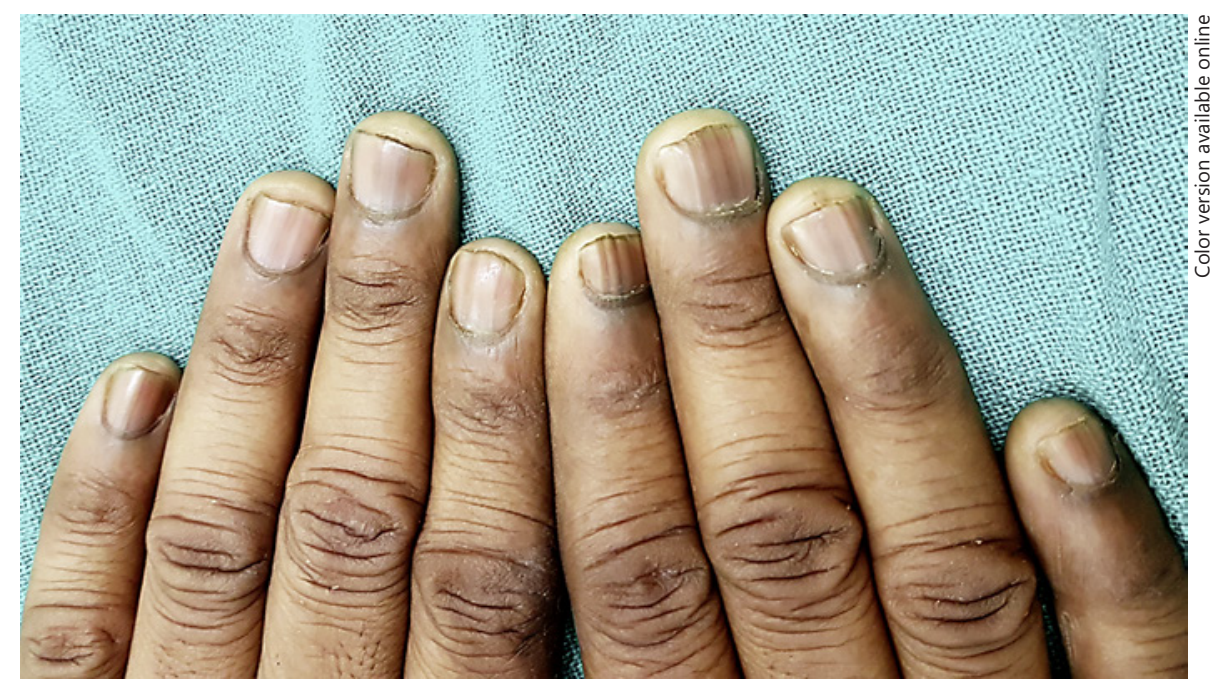

Fig. 2. Greyish black diffuse pigmentation in patient 2 .

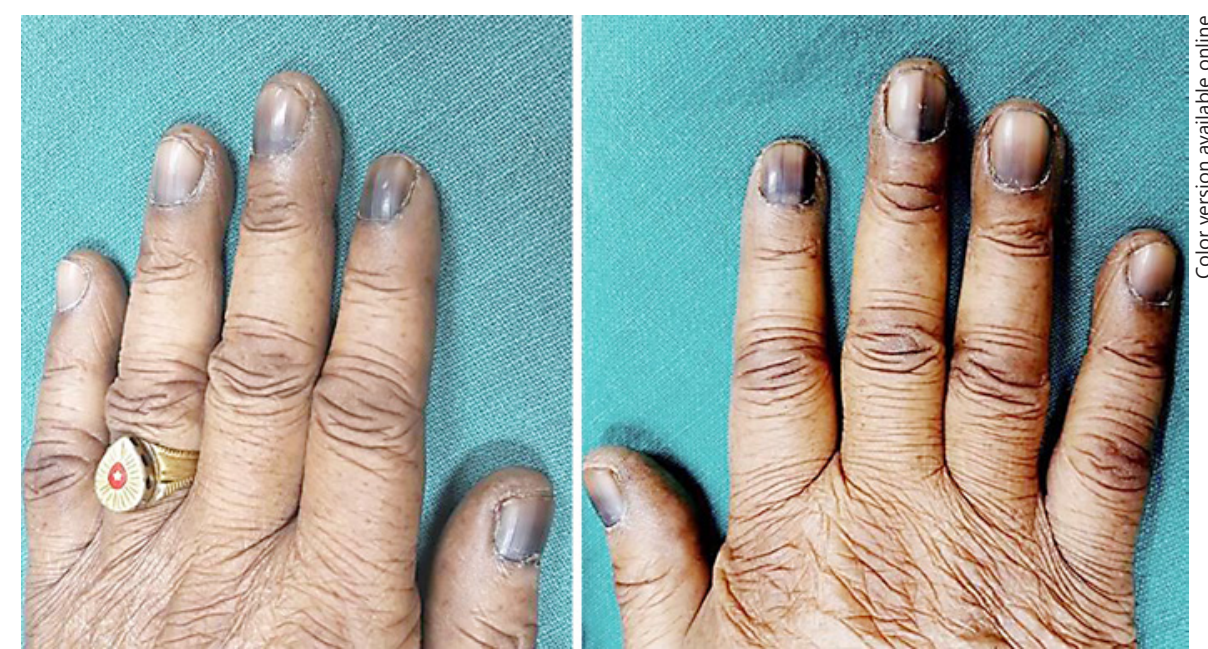

nosis can be established early in the acute phase of infection, within the first week, by reverse-transcription polymerase chain reaction.

Mucocutaneous manifestations have been reported to be significant and predominant findings in CHKV infection seen in about $35-50 \%$ of the patients [2-5]. There has been a number of studies from India detailing the nature of various mucocutaneous manifestations in CHKV infection [2-5]. Few skin lesions, like centrofacial or nose pigmentation, are very characteristic and specific and, thus, help in distinguishing the infection from its close differential of dengue fever as well as in the retrospective diagnosis of CHKV infection. All lesions resolve spontaneously, except pigmentary changes that may take months to resolve. We report 2 serologically confirmed cases of
$\mathrm{CHK}$ presenting with isolated diffuse nail pigmentation in the absence of any mucocutaneous involvement.

\section{Case Reports}

A 48-year-old man presented with asymptomatic diffuse pigmentation of all finger nails of approximately 2 weeks' duration. Examination revealed an otherwise healthy, well-built man with a greyish brown discoloration involving all fingernails. The pigmentation was accentuated along the longitudinal ridges that had been present in all nails for the past 3-4 years (Fig. 1). The skin overlying the distal digit of all fingers and the interphalangeal joints were also pigmented. The toenails showed diffuse pigmentation too, but it was less perceptible. The patient had a history of sudden-onset high-grade fever with severe joint and muscle pain that was diagnosed as CHK 2 weeks before the onset of nail pigmentation. 
A week later, a 56-year-old female patient was referred from the medicine department for sudden onset of greyish black diffuse pigmentation of all fingernails appearing within 2 weeks of the subsidence of CHKV infection. The pigmentation was more marked over the proximal half of the nail plates (Fig. 2).

There was no evidence of facial pigmentation or any other mucocutaneous involvement in either of the 2 patients. Both denied drug intake within the past 3 months except for acetaminophen for the CHK for about 3-4 weeks. Serology for IgM antibodies to $\mathrm{CHKV}$ infection was positive in both. Hematological and biochemical investigations, including serum vitamin $\mathrm{B}_{12}$ levels, were undertaken and were found to be normal in both patients. The course of CHKV infection was not debilitating with almost complete recovery within 2 weeks with minimal morbidity in the form of residual mild joint pain. The patients were counselled about the benign nature of nail pigmentation and the possibility of spontaneous recovery akin to that observed with the pigmented skin lesions associated with CHKV infection. Follow-up of both patients at 1 month did not reveal any change in the nail pigmentation.

\section{Discussion}

Protean mucocutaneous manifestations have been reported with $\mathrm{CHKV}$ infection. The commonest cutaneous manifestation is a maculopapular rash starting from the face and neck and subsequently becoming generalized [2]. It appears in 3-5 days and fades or resolves with desquamation in about 1 week. Other reported lesions include leg ulcers, purpuric lesions, targetoid lesions, confluent hemorrhagic vesiculobullous lesions, and peeling of skin resembling toxic epidermal necrolysis [2-4]. In addition, desquamation of the palms and soles, lichenoid lesions, and photosensitivity have also been described [2]. Mucosal lesions that resolve spontaneously after 7-10 days have been reported in $15-18 \%$ of the patients [4]. Characteristic pigmentation of the nose termed "chik sign" has been observed in most series appearing within 7-10 days of disease onset. The pigmentation tends to persist for 3-6 months and gradually resolves spontaneously $[2,4,5]$. The etiopathogenesis of pigmentation remains speculative. Hematoxylin and eosin-stained sections of pigmentary lesions have revealed diffuse hypermelanosis of the entire epidermis, intact basal layer with absent pigmentary incontinence, and mild perivascular infiltrate suggesting increased intra-epidermal melanin dispersion triggered by the virus [2]. Nail involvement is rare and has been described in a single case series [4]. Black lunula, subungual hemorrhage, and onychomadesis have been reported to coexist with other skin lesions [4]. The onset of diffuse melanonychia immediately following febrile illnesses of $\mathrm{CHK}$ in both patients and the established association of CHKV infection with pigmentary mucocutaneous changes suggest that the observed pigmentary nail changes can be attributed to $\mathrm{CHKV}$ infection. A similar pathogenetic mechanism as suggested for skin pigmentation may be extrapolated to melanonychia.

To the best of our knowledge, isolated nail pigmentation/melanonychia associated with $\mathrm{CHK}$ has not been described so far. Awareness of the possibility of this benign presentation shall obviate the employment of invasive diagnostic tests and aggressive treatment.

\section{Statement of Ethics}

Written informed consent has been obtained from both patients.

\section{Disclosure Statement}

The authors hereby declare to have no conflicts of interest regarding this paper.

\section{References}

Lo Presti A, Lai A, Cella E, Zehender G, Ciccozi M: Chikungunya virus, epidemiology, clinics and phylogenesis: a review. Asian Pac J Trop Med 2014;7:925-932.

2 Inamadar AC, Palit A, Sampagavi VV, Raghunath S, Deshmukh NS: Cutaneous manifestations of chikungunya fever: observations made during a recent outbreak in south India. Int J Dermatol 2008;47:154-159.

-3 Pakran J, George M, Riyaz N, Arakkal R, George S, Rajan U, et al: Purpuric macules with vesiculobullous lesions: a novel manifestation of chikungunya. Int J Dermatol 2011; 50:61-69.
4 Riyaz N, Riyaz A, Rahima, Abdul Latheef EN, Anitha PM, Aravindan KP, et al: Cutaneous manifestations of chikungunya during a recent epidemic in Calicut, north Kerala, south India. Indian J Dermatol Venereol Leprol 2010;76:671-676.

5 Bhat RM, Rai Y, Ramesh A, Nandakishore B, Sukumar D, Martis J, et al: Mucocutaneous manifestations of chikungunya fever: a study from an epidemic in coastal Karnataka. Indian J Dermatol 2011;56:290-294. 